\title{
Reeh-Schlieder Meets Newton-Wigner
}

\author{
Gordon N. Fleming* \\ Pennsylvania State University \\ *gnf1@earthlink.net
}

\begin{abstract}
The Reeh-Schlieder theorem asserts the vacuum and certain other states to be spacelike superentangled relative to local fields. This motivates an inquiry into the physical status of various concepts of localization. It is argued that a covariant generalization of NewtonWigner localization is a physically illuminating concept. When analyzed in terms of nonlocally covariant quantum fields, creating and annihilating quanta in Newton-Wigner localized states, the vacuum is seen to not possess the spacelike superentanglement that the Reeh-Schlieder theorem displays relative to local fields, and to be locally empty as well as globally empty. Newton-Wigner localization is then shown to be physically interpretable in terms of a covariant generalization of the center of energy, the two localizations being identical if the system has no internal angular momentum. Finally, some of the counterintuitive features of Newton-Wigner localization are shown to have close analogues in classical special relativity.

1. Introduction: Relativistic quantum field theory (QFT) presents us with a number of novel counterintuitive features, over and above those of general quantum theory which, for some time now, have been discussed from various philosophical perspectives. Among the novelties we have the breakdown of unitary equivalence between the basic dynamical variables of an interacting field with two distinct values of the interaction strength. This is known as Haag's theorem (Haag, '92, p.57, and references therein). As a consequence it is impossible to compare, in one and the same Hilbert space, how the field theoretic system will evolve with and without the interaction turned on. We have the property of the vacuum state (and others), called Superentangled by Clifton et al (98), which allows any part of the Hilbert space to be reached by operations on the vacuum state with operators constructed within any, arbitrarily small space-time region. This is known as the Reeh-Schlieder theorem (Reeh and Schlieder, ' 61 ). And, thirdly, we have the presence of Rindler quanta in the vacuum state(Unruh, '76; Birrell and Davies, '82, p.109; Unruh, '90; Wald, '94, Ch.5 and references therein).
\end{abstract}

Invariably the term 'counterintuitive', as used here, refers to seemingly strange and bizarre differences between the quantum domain and what we have come to expect on the basis of our long term exposure to classical physics. Accordingly, one might well argue that we must simply bite the bullet of recognizing classical physics as a poorer 
approximation to reality than quantum theory. We must shed our classical intuitions, painful as that experience may be, and get on with the business of building a quantum intuition. That quantum intuition will then serve us well until a still better theoretical scheme than quantum theory comes along and the painful process must be repeated. Indeed, physicists are trained to do just that (the training doesn't always take) and, as a physicist, I am very sympathetic to that policy. However, in the interests of developing deeper understanding and philosophically sophisticated interpretations, it is valuable to study, in some detail, the relationships between the counterintuitive quantum features and the corresponding or analogous classical features. A lurking danger in such endeavors is that of unconsciously lapsing into regarding only classical accounts or accounts consistent with a classical ontology as constituting real understanding, and, thus, trying to explain quantum strangeness along classical lines. Conciously embracing such a view is not a danger, but simply a philosophically conservative and, I think, naïve position. Having noted the lurking danger, the effort should be undertaken.

In this presentation there is far too little space to consider all the counterintuitive features of QFT that I've mentioned. I have, therefore, chosen to focus on the Reeh-Schlieder theorem and the novel perspective on that theorem provided by Newton-Wigner fields (Fulling, '89, pp.54-56), which create and annihilate quanta in Newton-Wigner position eigenstates (Newton '49). Hence my title. To keep kinematical considerations as simple as possible, I will discuss scalar fields only, with their associated spinless quanta. A comment on how matters generalize to higher rank fields will be made at the end. To permit the least questionable use of the somewhat more familiar language of heuristic QFT, and to disentangle the Reeh-Schlieder issue from the dynamical questions surrounding Haag's theorem, I will consider only a non-interacting field. This last is a severe limitation on our territory. But it enables us to shine a brighter light upon it.

A few years ago Michael Redhead (1995a, b) presented very illuminating analyses of the implications of the Reeh-Schlieder theorem and extended our awareness of the EPR type correllations existing in the bounded energy states of relativisitc QFT. In the process, however, Redhead argued that an important ingredient in understanding these features for the vacuum was the impossibility, in the free field case, of converting the global statement of the absence of quanta in the vacuum as a whole, at any time, into local statements of the absence of quanta in bounded regions of space at any time. Unlike the case in non-relativistic QFT, the most obvious candidate for the number density operator at a point fails to commute with itself at distinct points of space. Thus one can not, simultaneously, claim the absence of quanta in two disjoint volumes, even in the vacuum. He then argued against the counterexample provided by the number density operators from Newton-Wigner fields on the grounds of the violation of covariance and physical inconsistency in the account of localization.

I believe this latter argument to be misguided and will show below that a natural generalization of the Newton-Wigner fields restores covariance, albeit not local covariance, and that interesting implications rather than inconsistency is found in the attendent account of localization. 
In passing, Rindler quanta will be briefly mentioned as illustrating Reeh-Schlieder superentanglement.

2. The Reeh-Schlieder Theorem: We begin the discussion by considering the relevent state vectors. State vectors represent the relationship of a physical state of affairs to a frame of reference or space-time coordinate system. In QFT the defining characteristic of the vacuum state is the absence of measureable structure that would differ from one inertial frame to another, i.e. the physical state of affairs has the same relationship to every inertial frame. Thus, if $\mid \Omega>$ represents the vacuum in the frame $F$ and $\mid \Omega^{\prime}>$ represents the vacuum in the frame $F^{\prime}$, then

$$
\left|\Omega^{\prime}>=\right| \Omega>
$$

This being the case, it then follows that the total 4-momentum operator, $\hat{\mathrm{P}}^{\mu}$, and the total generalized angular momentum (6-momentum) operator, $\hat{\mathrm{M}}^{\mu \nu}$, since these operators produce the changes in a state vector when one changes the reference frame by infinitesimal Poincare' transformations, have the effect,

$$
\hat{\mathrm{P}}^{\mu}\left|\Omega>=0=\hat{\mathrm{M}}^{\mu v}\right| \Omega>
$$

In other words the QFT vacuum, quite in keeping with classical intuition, is devoid of total energy, momentum and generalized angular momentum.

The other state vectors to which the Reeh-Schlieder theorem applies are those for which the energy spectrum is bounded. This means there exists an energy value, $\mathrm{E}_{0}$, such that

$$
<\Psi\left|\left(\hat{\mathrm{P}}^{0}\right)^{\mathrm{n}}\right| \Psi>\leq \mathrm{E}_{0}{ }^{\mathrm{n}}<\Psi \mid \Psi>
$$

for all $\mathrm{n}$. Such state vectors are densely distributed through the state space.

Next we turn to the operators which figure in the Reeh-Schlieder theorem. Our 'operator' valued field will be denoted by, $\hat{\phi}(\mathrm{x})$, and to allow the anti-quanta of the field to be distinct from the quanta, the field will be distinct from its adjoint, $\hat{\phi}^{\dagger}(\mathrm{x})$. Among the properties of the field that will play important roles in our discussion are:

(1.) Locally covariant transformation rule (in this case, scalar),

$$
<\Psi^{\prime}\left|\hat{\phi}\left(\mathrm{x}^{\prime}\right)\right| \Psi^{\prime}>=<\Psi|\hat{\phi}(\mathrm{x})| \Psi>
$$

for any $|\Psi\rangle$, where, $\left|\Psi^{\prime}\right\rangle$ is obtained from $\mid \Psi>$ by unitary

representatives of Poincare' transformations, 


$$
\left|\Psi^{\prime}>=\hat{\mathrm{U}}(\Lambda, \mathrm{a})\right| \Psi>
$$

(2.) Microcausality,

$$
\left[\hat{\phi}\left(x^{\prime}\right), \hat{\phi}(x)\right]=\left[\hat{\phi}\left(x^{\prime}\right), \hat{\phi}^{\dagger}(x)\right]=0,
$$

for $\mathrm{x}$ and $\mathrm{x}^{\prime}$ relatively space-like.

(3.) Free field equation of motion,

$$
\left(\partial^{\mu} \partial_{\mu}+\kappa^{2}\right) \hat{\phi}(x)=0 .
$$

(4.) Canonical commutation relations,

$$
\begin{gathered}
{\left[\hat{\phi}\left(\mathrm{x}^{0}, \mathbf{x}^{\prime}\right), \frac{\partial}{\partial \mathrm{x}^{0}} \hat{\phi}^{\dagger}\left(\mathrm{x}^{0}, \mathbf{x}\right)\right]=\mathrm{i} \delta^{3}\left(\mathbf{x}^{\prime}-\mathbf{x}\right),} \\
{\left[\hat{\phi}\left(\mathrm{x}^{0}, \mathbf{x}^{\prime}\right), \frac{\partial}{\partial \mathrm{x}^{0}} \hat{\phi}\left(\mathrm{x}^{0}, \mathbf{x}\right)\right]=0 .}
\end{gathered}
$$

Features (3.) and (4.) do not play a role in the proof of the theorem, as stated below, but if they hold, as they do in our example, the theorem can be strengthened in a manner we will mention.

Now consider any bounded open region, $O$, of space-time, and the set, $\mathrm{C}^{\infty}(O)$, of all smooth, real functions on space-time which vanish outside $O$. For every $\mathrm{f} \in \mathrm{C}^{\infty}(O)$, define

$$
\hat{\phi}(f):=\int d^{4} x f(x) \hat{\phi}(x) .
$$

Finally build the operator algebra, $\mathbf{A}(O)$, by taking all possible combinations of adjoints, products and sums of all the $\hat{\phi}(\mathrm{f})$. The theorem is now easily stated:

Reeh-Schlieder theorem: For any such region, $O$, the set of vectors, $\mathbf{A}(O) \mid \Omega>$, obtained by applying all the operators in $\mathbf{A}(O)$ to the vacuum state vector is dense in the Hilbert space, $\boldsymbol{H}$, of state vectors. The same is true of $\mathbf{A}(O) \mid \Psi>$, where $\mid \Psi>$ is any state vector of bounded energy,

The technical term for this property of the vacuum state and the bounded energy states is that they are 'cyclic' relative to $\mathbf{A}(O)$. 
The strengthening of the theorem that can be achieved if the relationships (3.) and (4.) are invoked is that the open 4-dimensional regions, $O$, and the associated algebras, $\mathbf{A}(O)$, can be relaced by open 3-dimensional regions of space at a single time, $\left(\mathrm{x}^{0}, O\right)$, and their associated algebras, $\mathbf{A}\left(\mathrm{x}^{0}, O\right)$. By allowing interactions in the evolution of the field one loses the assurance that smearing the fields in 3-dimensions is sufficient to handle the singular nature of the field.

Now just what is counterintuitive about this theorem? Well, it is common practice to regard the operators in $\mathbf{A}(O)$ as representing actions or operations that can be performed within $O$, and, if an operator is self adjoint, as representing a quantity that can be measured by actions taken within $O$. No claim is made, of course, that the corresponding actions for a given operator would be simple, or even within current technological capacities. Only that such actions are possible in principle. But if so the theorem is amazing! For suppose $|\Theta\rangle$ is a state vector describing a state of affairs in which the only deviation from vacuum properties occurrs within the union of the interior of the future and past lightcone envelopes of a region, $O^{\prime}$, that is space-like separated from $O$. Then according to Reeh-Schlieder actions can be performed on the vacuum within $O$ which will produce a state vector as close to $\mid \Theta>$ in the Hilbert space as you like. And $O$ can be as small as you like, and as far, space-like wise, from $O^{\prime}$ as you like.

Corollary to the Reeh-Schlieder Theorem: This is not all! From the microcausality condition it follows that when $O$ and $O^{\prime}$ are relatively space-like every member of $\mathbf{A}(O)$ commutes with every member of $\mathbf{A}\left(O^{\prime}\right)$ and from this it follows that no non-zero operator in any such algebra can annihilate the vacuum state. The technical term designating this property is that the vacuum is 'separating' with respect to the algebras.

But this means there are no pure annihilation operators in any $\mathbf{A}(O)$ since,

$$
\hat{\mathrm{a}} \in \mathbf{A}(O) \text {, and } \hat{\mathrm{a}} \mid \Omega>=0 \quad \Rightarrow \quad \hat{\mathrm{a}}=0 \text {, }
$$

i.e. no annihilation operators for anything! And, by conjugation, no creation operators either. In other words, countable systems can not be added to the vacuum by local operations. Nor, however added, can they be removed by local operations! 


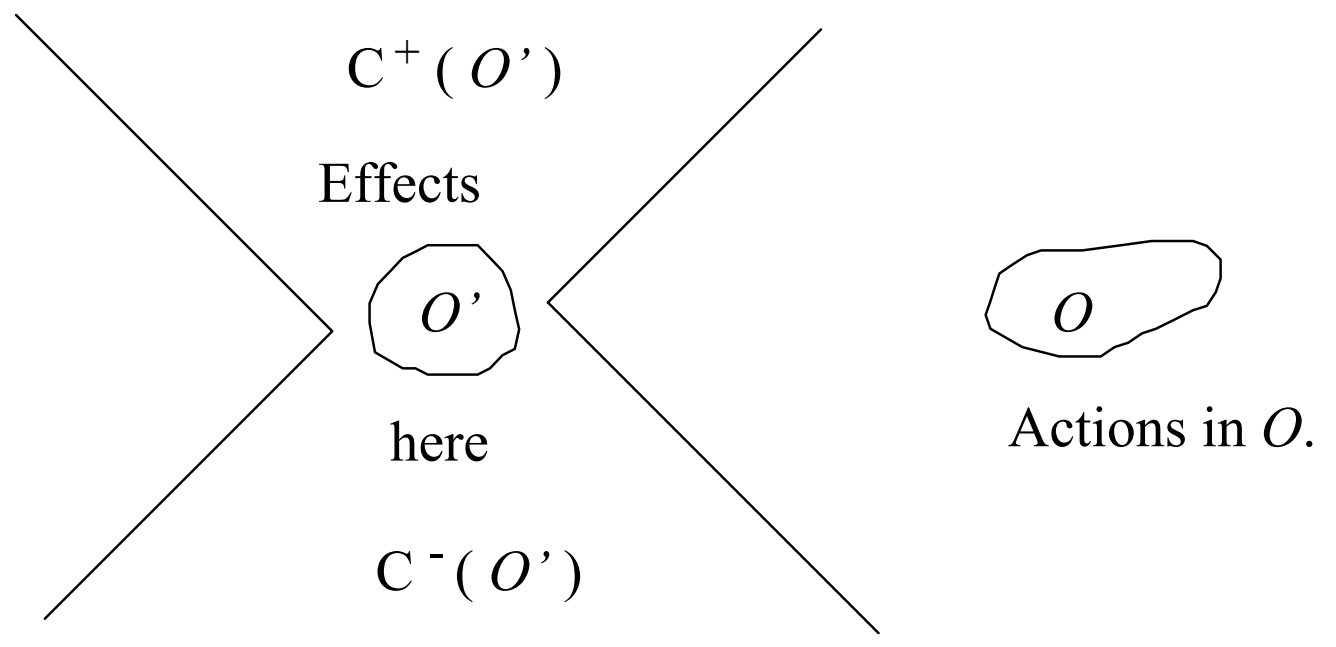

\section{Fig. 1: Space-like separated effects allowed by Reeh-Schlieder}

The common interpretation is easily subject to criticism. For if you and the necessarry aparatus are in $O$, to perform the actions in question, it is not clear in what sense they are being performed on the vacuum! This criticism is not so easily brought if the state is merely of bounded energy, rather than the vacuum. You and your aparatus might well be part of a state of bounded energy. But quite apart from all this anthropomorphising, it is remarkable that any state can have enough structure within an arbitrarily small region, $O$, to enable even the mathematical reconstituting of essentially the whole state space. This is the kind of feature that gives rise to the term 'superentangled' of Clifton et al. For the vacuum state to be such a state was a great, counterintuitive, surprise.

Interestingly enough, an explicit, albeit formal, expression for the entanglment in the vacuum between two space-like separated regions, $O$ and $O^{\prime}$, can be constructed employing the creation operators obtained from a Fourier transform of the field in Rindler-anti-Rindler coordinates. The possibility depends on the fact that between any two space-like separated regions of Minkowski space-time one can find intersecting hyperplanes, tangent to a light cone, which define the boundaries of a rindler-anti-Rindler coordinate system. The physical, or Minkowski vacuum state, $\mid \Omega>$, is then expressed in terms of Rindler and anti- Rindler quanta creation operators acting on the Rindler-antiRindler vacuum state, $\mid 0_{\overline{\mathrm{R}}}, 0_{\mathrm{R}}>$ (Fig. 2) 


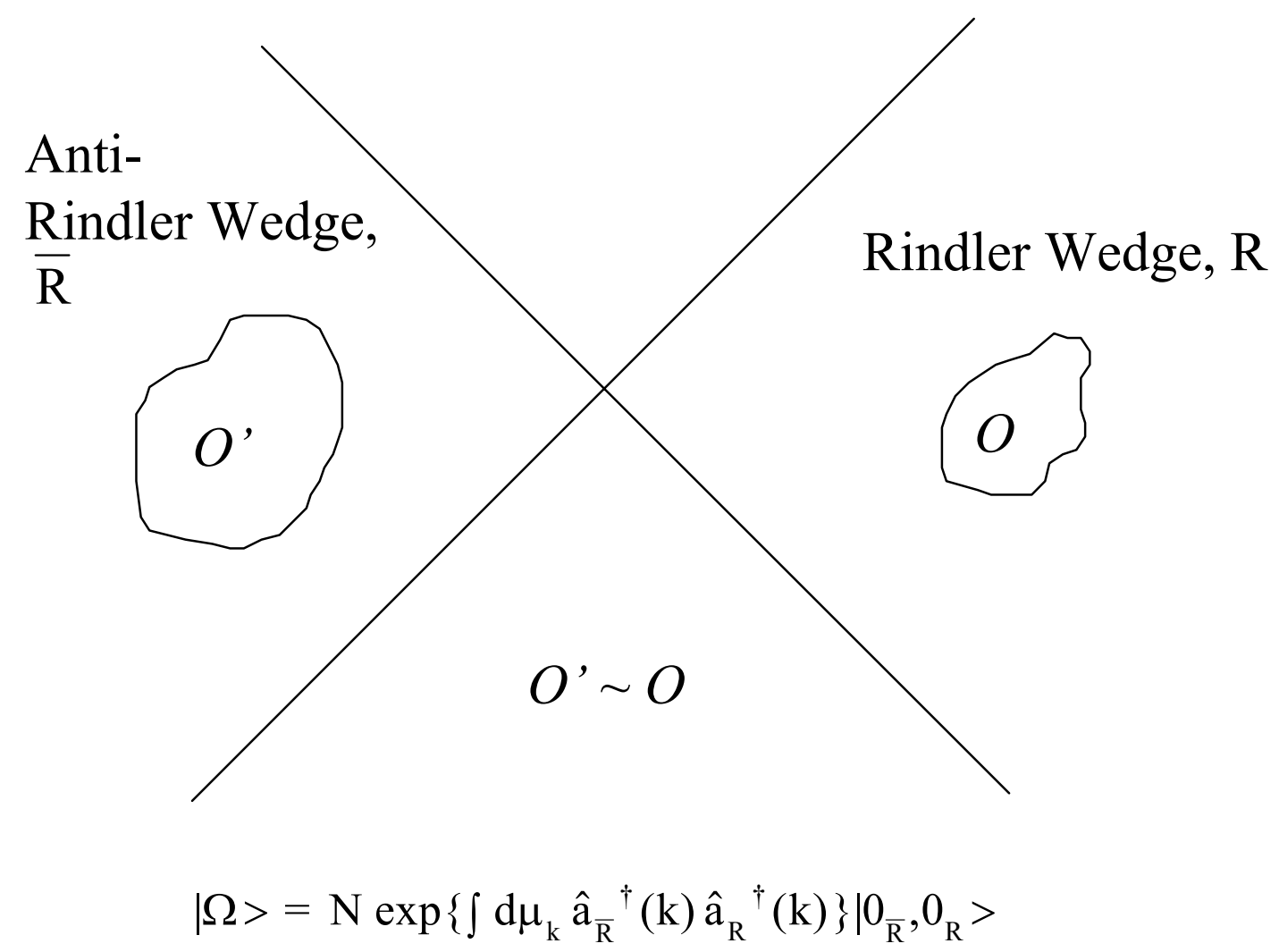

Fig. 2: Vacuum entanglement via Rindler quantization

Some comment may be useful here. The operators of $\mathbf{A}(O)$ are formal functionals of the Rindler operators, $\hat{a}_{\mathrm{R}}(\mathrm{k})$, and their adjoints only. No antiRindler operators are involved. The operators of $\mathbf{A}\left(O^{\prime}\right)$, on the other hand, are formal functionals of the anti-Rindler operators, $\hat{a}_{\overline{\mathrm{R}}}(\mathrm{k})$, and their adjoints, alone. The equation in the figure, therefore, expresses, in terms of the Rindler-antiRindler vacuum, the entanglement in the Minkowski vacuum state between the region $O$ and the space-like separated region $O^{\prime}$ and its causal envelope.

Furthermore, suppose $\hat{\Pi}$ is a projection operator in $\mathbf{A}(O)$, i.e. $\hat{\Pi}$ represents a possible outcome of some local measurement in $O$. Then, since,

$$
P(\hat{\Pi} / \Omega)=<\Omega|\hat{\Pi}| \Omega>=\|\hat{\Pi} \mid \Omega>\|^{2},
$$

where $P$ denotes probability, we have,

$$
P(\hat{\Pi} / \Omega)=0 \Rightarrow \hat{\Pi} \mid \Omega>=0 \Rightarrow \hat{\Pi}=0
$$


Thus every possible outcome $(\hat{\Pi} \neq 0$ ) of a local measurement in $O$ has a non-zero probability of occurring in the vacuum! Again a very counterintuitive result. Not exactly paradoxical, to be sure, but is it, perhaps, time to question the meaning of being localized?!

3. Knight-Licht Strict Localization: Many concepts of localization have been proposed over the years. We will consider two. The first, proposed by Knight ('61) and Licht ('63) is very much in the spirit of the Local Algebra approach to QFT in which the ReehSchlieder theorem is couched. It has, however, some seriously objectionable features.

Denoting the causal future, + , and causal past, -, of a Space-time region, $O^{\prime}$, by $\mathrm{C}^{ \pm}\left(O^{\prime}\right)$, we say that the state vector, $|\Psi\rangle$, describes systems and processes strictly localized within $U_{O^{\prime}}:=\mathrm{C}^{+}\left(O^{\prime}\right) \cup \mathrm{C}^{-}\left(O^{\prime}\right)$, iff

$$
<\Psi|\hat{\mathrm{A}}| \Psi>=<\Omega|\hat{\mathrm{A}}| \Omega>
$$

for any $\hat{\mathrm{A}} \in \mathbf{A}(O)$, with $O$ completely outside of $U_{O}$, i.e. $O^{\prime}$ is space-like relative to $O$. It's very easy to build such states. For example,

$$
|\Psi>:=\exp [\mathrm{i} \hat{\mathrm{B}}]| \Omega>
$$

is such a state if $\hat{\mathrm{B}}=\hat{\mathrm{B}}^{\dagger} \in \mathbf{A}\left(O^{\prime}\right)$. Unfortunately, it turns out that strictly localized states can not contain only a finite number of quanta. At best, they can approach finite quanta states arbitrarily closely. Conceptually more serious is that if $|\Psi\rangle$ is strictly localized within $U_{O}$ and $\mid \Phi>$ is strictly localized within $U_{O^{\prime}}$, where $O$ and $O^{\prime}$ are relatively spacelike, the state vectors need not be orthogonal. This is distressing since, in any inertial reference frame, there would be time intervals during which the non-vacuum physical state of affairs would be spatially disjoint in $|\Psi\rangle$ and $|\Phi\rangle$. The non-zero inner product between the state vectors would then have to be interpreted as proportional to a probability amplitude for finding the system/processes confined to one of the spatial regions given that, simultaneously, the system/processes are confined to the other. Accepting counterintuitive consequences of an introduced concept is one thing. Accepting consequences that undermine the very concept one is trying to construct is quite another thing. We turn to another localization concept.

4. Newton-Wigner Localized States: One might well ask why a simple appeal to the decomposition of the field into positive and negative frequency parts,

$$
\hat{\phi}(\mathrm{x})=\hat{\phi}^{(+)}(\mathrm{x})+\hat{\phi}^{(-)}(\mathrm{x})
$$

doesn't suffice to provide a satisfactory localization concept? Especially in the case of free fields, this decomposition is unequivocally interpretable as the separation of the local 
space-time field, $\hat{\phi}(\mathrm{x})$, into the part, $\hat{\phi}^{(+)}(\mathrm{x})$, which removes (in a manner associated with the space-time point, $x$ ) a single quanta from any state vector it's applied to (if they're there to be removed), and and the part, $\hat{\phi}^{(-)}(\mathrm{x})$, which similarly adds a single anti-quanta. That being the case, why isn't it natural to identify

$$
\hat{\phi}^{(+)}(\mathrm{x})^{\dagger}|\Omega>:=|(+), \mathrm{x}>, \quad \text { and } \quad \hat{\phi}^{(-)}(\mathrm{x})|\Omega>:=|(-), \mathrm{x}>
$$

as the states of single quanta and anti-quanta, respectively, localized at the space-time point, $x$ ? The reason for not doing so is exactly the same as the most serious objection to Knight-Licht Strict Localization, i.e., the absence of orthogonality. Unlike the local field, $\hat{\phi}(\mathrm{x})$, itself, the positive and negative frequency parts do not commute (with their adjoints ) at space-like separation and, consequently, the previously defined states are not orthogonal for space-like separation, i.e., for $\mathrm{x} \sim \mathrm{x}^{\prime}$,

$$
<( \pm), x \mid( \pm), x^{\prime}>\neq 0 \text {. }
$$

So, once again, localization (of something) here, now, would not be incompatible with localization (of the same thing) there, now. But that is incompatible with the concept of localization one is trying to articulate.

In fact this specific problem of orthogonality of putatively localized states had already been 'solved' in 1949 by Newton and Wigner (NW). The context in which they worked was the single particle state space but it is straightforward to reformulate their results in QFT (Fuller, '89, p.54). Again one starts with the positive and negative frequency parts of the local field, but now one modifies them with an integral transform,

$$
\hat{\phi}_{\mathrm{NW}}^{\dagger}\left(\mathrm{x}^{0}, \mathbf{x}\right):=\left(\sqrt{2 \mathrm{R}} \hat{\phi}^{(+)}{ }^{\dagger}\right)\left(\mathrm{x}^{0}, \mathbf{x}\right), \quad \hat{\bar{\phi}}_{\mathrm{NW}}^{\dagger}\left(\mathrm{x}^{0}, \mathbf{x}\right):=\left(\sqrt{2 \mathrm{R}} \hat{\phi}^{(-)}\right)\left(\mathrm{x}^{0}, \mathbf{x}\right),
$$

where

$$
\mathrm{R}:=\sqrt{\kappa^{2}-\left(\frac{\partial}{\partial \mathbf{x}}\right)^{2}}
$$

as mentioned, is not the spatially local differential operator that the compact expression suggests. In fact it is a highly non-local integral transform. Nevertheless, the state vectors (with corresponding relations holding for the anti-quanta),

$$
\left|\mathrm{x}^{0}, \mathbf{x}>_{\mathrm{NW}}:=\hat{\phi}_{\mathrm{NW}}^{\dagger}\left(\mathrm{x}^{0}, \mathbf{x}\right)\right| \Omega>
$$

satisfy the orthogonality condition,

$$
{ }_{\mathrm{NW}}<\mathrm{x}^{0}, \mathbf{x} \mid \mathbf{x}^{0}, \mathbf{x}^{\prime}>_{\mathrm{NW}}=\delta^{3}\left(\mathbf{x}-\mathbf{x}^{\prime}\right),
$$


while the fields satisfy the equal time commutation relations,

$$
\begin{aligned}
& {\left[\hat{\phi}_{\mathrm{NW}}\left(\mathrm{x}^{0}, \mathbf{x}\right), \hat{\phi}_{\mathrm{NW}}^{\dagger}\left(\mathrm{x}^{0}, \mathbf{x}^{\prime}\right)\right]=\delta^{3}\left(\mathbf{x}-\mathbf{x}^{\prime}\right),} \\
& {\left[\hat{\phi}_{\mathrm{NW}}\left(\mathrm{x}^{0}, \mathbf{x}\right), \hat{\phi}_{\mathrm{NW}}\left(\mathrm{x}^{0}, \mathbf{x}^{\prime}\right)\right]=0,}
\end{aligned}
$$

and evolve according to,

$$
\mathrm{i} \partial^{0} \hat{\phi}_{\mathrm{NW}}\left(\mathrm{x}^{0}, \mathbf{x}\right)=\mathrm{R} \hat{\phi}_{\mathrm{NW}}\left(\mathrm{x}^{0}, \mathbf{x}\right) .
$$

Thus, even for multi quanta-anti-quanta states, a coherent notion of being localized here now, i.e. in a definite region of space at a definite time seemed to be available. All was not well, however. While the NW localized states transformed as expected under the Euclidean group of rotations, $\Lambda_{\mathrm{R}}$, and spatial translations,

$$
\hat{\mathrm{U}}\left(\Lambda_{\mathrm{R}},(0, \mathbf{a})\right)\left|\mathrm{x}^{0}, \mathbf{x}>_{\mathrm{NW}}=\right| \mathrm{x}^{0}, \Lambda_{\mathrm{R}} \mathbf{x}+\mathbf{a}>_{\mathrm{NW}},
$$

they did not enjoy locally covariant transformation rules under any transformation involving a Lorentz boost, $\Lambda_{\mathrm{B}}$. At the field theoretic level this takes the form,

$$
\forall\left(\mathrm{x}^{0}, \mathbf{x}^{\prime}\right), \quad \hat{\phi}_{\mathrm{NW}}\left(\mathrm{x}^{0}{ }^{\prime}, \mathbf{x}^{\prime}\right) \neq \hat{\mathrm{U}}\left(\Lambda_{\mathrm{B}}, \mathrm{a}\right) \hat{\phi}_{\mathrm{NW}}\left(\mathrm{x}^{0}, \mathbf{x}\right) \hat{\mathrm{U}}^{\dagger}\left(\Lambda_{\mathrm{B}}, \mathrm{a}\right)
$$

i.e., a boost of the NW field at a given position and moment is not equal to the NW field at any position and moment! Furthermore, as follows from the evolution equation for the field, the NW localized states have a superluminal contribution to their evolution, (Fleming, '65; Ruijsenaars, '81; Hegerfeldt, '85) i.e.

$$
\mathrm{NW}_{\mathrm{NW}}<\mathrm{x}^{0}, \mathbf{x} \mid \mathrm{x}^{0}{ }^{\prime}, \mathbf{x}^{\prime}>_{\mathrm{NW}} \neq 0,
$$

for space-like separated $\mathrm{x}$ and $\mathrm{x}^{\prime}$ if $\mathrm{x}^{0} \neq \mathrm{x}^{0}$. These results ultimately dismayed Wigner ('83, pp.310-313) and lead to Redheads' assessment mentioned earlier. But we will shortly see that these negative judgements are premature. By recognizing the NW states and the fields that create them as members of a larger family we will recover fully covariant transformation properties (Fleming, '66, p.1978; '96). But why make the effort?! What is it about the NW fields that is relevant to the assessment of the ReehSchlieder theorem and the structure of the vacuum?

5. The Vacuum Relative to the NW Fields: Here we exploit the free evolution of the NW fields and consider the algebras, $\mathbf{A}_{\mathrm{NW}}\left(\mathrm{x}^{0}, O\right)$, generated by polynomials in the 3 dimensionally smeared fields,

$$
\hat{\phi}_{\mathrm{NW}}\left(\mathrm{x}^{0}, \mathrm{f}\right):=\int \mathrm{d}^{3} \mathrm{x} f(\mathbf{x}) \hat{\phi}_{\mathrm{NW}}\left(\mathrm{x}^{0}, \mathbf{x}\right)
$$


and

$$
\hat{\bar{\phi}}_{\mathrm{NW}}\left(\mathrm{x}^{0}, \mathrm{f}\right):=\int \mathrm{d}^{3} \mathrm{x} f(\mathbf{x}) \hat{\bar{\phi}}_{\mathrm{NW}}\left(\mathrm{x}^{0}, \mathbf{x}\right) .
$$

It turns out that the vacuum state is not cyclic nor separating with respect to $\mathbf{A}_{\mathrm{Nw}}\left(\mathrm{x}^{0}, O\right)$ for any $\left(\mathrm{x}^{0}, O\right)$. Separation is trivially lost since each $\mathbf{A}_{\mathrm{NW}}\left(\mathrm{x}^{0}, O\right)$ contains the annihilating operators, $\hat{\phi}_{\mathrm{NW}}\left(\mathrm{x}^{0}, \mathrm{f}\right)$ and $\hat{\bar{\phi}}_{\mathrm{NW}}\left(\mathrm{x}^{0}, \mathrm{f}\right)$, for any smooth function, $\mathrm{f}$, with support confined in $O$. Cyclicity is then lost since $\mathbf{A}_{\mathrm{NW}}\left(\mathrm{x}^{0}, O\right)$ is a subset of the commutant of $\mathbf{A}_{\mathrm{NW}}\left(\mathrm{x}^{0}, O^{\prime}\right)$ for any $O^{\prime}$ disjoint from $O$. In fact the vacuum is a product state relative to the NW fields in the same way that Redhead indicated the non-relativistic vacuum was, relative to the Schroedinger field operator. Thus taking,

$$
\hat{\mathrm{n}}_{\mathrm{NW}}\left(\mathrm{x}^{0}, \mathbf{x}\right):=\hat{\phi}_{\mathrm{NW}}^{\dagger}\left(\mathrm{x}^{0}, \mathbf{x}\right) \hat{\phi}_{\mathrm{NW}}\left(\mathrm{x}^{0}, \mathbf{x}\right)
$$

as the definition of the number density operator for quanta ( and a corresponding definition for anti-quanta) we do have, for $\mathbf{x} \neq \mathbf{x}^{\prime}$,

$$
\left[\hat{\mathrm{n}}_{\mathrm{NW}}\left(\mathrm{x}^{0}, \mathbf{x}\right), \hat{\mathrm{n}}_{\mathrm{NW}}\left(\mathrm{x}^{0}, \mathbf{x}^{\prime}\right)\right]=0,
$$

and so the vacuum can, and does, simultaneously satisfy,

$$
\hat{\mathrm{n}}_{\mathrm{NW}}\left(\mathrm{x}^{0}, \mathbf{x}\right)\left|\Omega>=0=\hat{\mathrm{n}}_{\mathrm{NW}}\left(\mathrm{x}^{0}, \mathbf{x}^{\prime}\right)\right| \Omega>
$$

i.e. the vacuum is devoid of quanta (and anti-quanta) at every NW position at every time! Note that, unlike the Rindler-anti-Rindler quanta that were referred to earlier to display the Superentanglement of the vacuum, the quanta we're dealing with now are the garden variety kind. The same quanta and anti-quanta created and annihilated by the local field, $\hat{\phi}(\mathrm{x})$. It is only our way of assessing where they are that has been modified!

So, relative to local fields the vacuum has the Reeh-Schlieder property and can be said to be devoid of quanta only globally, not locally. On the other hand, relative to NW fields the vacuum does not have the Reeh-Schlieder property and is devoid of quanta locally as well as globally. Now it is clear why it would be worthwhile to see the NW fields as covariant structures.

6. Generalizing the NW Fields to Covariant Fields: For our present purpose it is useful to express $\hat{\phi}_{\mathrm{NW}}\left(\mathrm{x}^{0}, \mathbf{x}\right)$ and $\hat{\bar{\phi}}_{\mathrm{NW}}\left(\mathrm{x}^{0}, \mathbf{x}\right)$ in terms of the local field, $\hat{\phi}(\mathrm{x})$, itself, rather than in terms of its, already non-local, positive and negative frequency parts. We have,

$$
\hat{\phi}_{\mathrm{NW}}\left(\mathrm{x}^{0}, \mathbf{x}\right)=\frac{1}{\sqrt{2}}\left\{\mathrm{R}^{1 / 2} \hat{\phi}+\mathrm{i} \mathrm{R}^{-1 / 2} \partial^{0} \hat{\phi}\right\}\left(\mathrm{x}^{0}, \mathbf{x}\right),
$$




$$
\hat{\bar{\phi}}_{\mathrm{NW}}\left(\mathrm{x}^{0}, \mathbf{x}\right)=\frac{1}{\sqrt{2}}\left\{\mathrm{R}^{1 / 2} \hat{\phi}-\mathrm{iR} \mathrm{R}^{-1 / 2} \partial^{0} \hat{\phi}\right\}^{\dagger}\left(\mathrm{x}^{0}, \mathbf{x}\right)
$$

In this way we see just where the operator, $\mathrm{R}$, introduces non-locality. Reminding ourselves that $\mathrm{R}$ is a spatial integral transform, i.e. for some non-trivial $\mathrm{r}(\mathbf{x}-\mathbf{y})$,

$$
(\mathrm{R} \hat{\phi})\left(\mathrm{x}^{0}, \mathbf{x}\right):=\int \mathrm{d}^{3} \mathrm{y} \mathrm{r}(\mathbf{x}-\mathbf{y}) \hat{\phi}\left(\mathrm{x}^{0}, \mathbf{y}\right)
$$

we see that the NW fields are not merely associated with a single point of space-time, but with the whole of space at a particular time as well. It is then not surprising that they do not transform into one another under Lorentz boosts. For the whole of space at a single time in one inertial frame is a very different slice of space-time than the whole of space at any single time in another relatively moving inertial frame. Just as local fields, expressed in terms of the coordinates of distinct reference frames, are kinematically related only when the two sets of coordinates refer to the same space-time point, so our non-local NW fields will be kinematically related to fields in relatively moving frames that refer to one and the same pair of point of space-time and slice of space-time. But, to repeat, an instantaneous slice of space-time in one inertial frame is not an instantaneous slice in a relatively moving inertial frame. So, to find the kinematically related fields we must extend our definition of the NW fields to include fields similarly related to noninstantaneous slices of space-time, i.e. to arbitrary space-like hyperplanes.

To do this, all that is required is to appropriately alter $\mathrm{R}$ and $\partial^{0}$ which occur in the definitions. Thus we introduce (Fleming, '96) the future pointing, time-like, unit 4-vector, $\eta^{\mu}$, and define,

$$
\mathrm{D}_{\eta}^{\mu}:=\partial^{\mu}-\eta^{\mu}(\eta \partial)
$$

and

$$
\mathrm{R}_{\eta}:=\sqrt{\kappa^{2}+\mathrm{D}_{\eta}^{2}}=\sqrt{\kappa^{2}+\partial^{\mu} \partial_{\mu}-(\eta \partial)^{2}}
$$

and, finally,

$$
\begin{gathered}
\hat{\phi}_{\mathrm{NW}}(\eta, \mathrm{x}):=\frac{1}{\sqrt{2}}\left\{\mathrm{R}_{\eta}{ }^{1 / 2} \hat{\phi}+\mathrm{i} \mathrm{R}_{\eta}{ }^{-1 / 2}(\eta \partial) \hat{\phi}\right\}(\eta, \mathrm{x}), \\
\hat{\bar{\phi}}_{\mathrm{NW}}(\eta, \mathrm{x}):=\frac{1}{\sqrt{2}}\left\{\mathrm{R}_{\eta}{ }^{1 / 2} \hat{\phi}-\mathrm{i} \mathrm{R}_{\eta}{ }^{-1 / 2}(\eta \partial) \hat{\phi}\right\}^{\dagger}(\eta, \mathrm{x}) .
\end{gathered}
$$

The generalized operator, $\mathrm{R}_{\eta}$, is an integral operator over a hyperplane consisting of points with coordinates, $y$, for which $\eta y$ is fixed. For $\eta^{\mu} \neq(1, \mathbf{0})$, this is a noninstantaneous hyperplane and as $\eta$ varies over its possible values, all possible space-like 
hyperplane orientations are included. And, indeed, as expected, under the unitary representation of the inhomogeneous Lorentz group, $\hat{U}(\Lambda, a)$, we now have

$$
\hat{\mathrm{U}}(\Lambda, \mathrm{a}) \hat{\phi}_{\mathrm{NW}}(\eta, \mathrm{x}) \hat{\mathrm{U}}^{\dagger}(\Lambda, \mathrm{a})=\hat{\phi}_{\mathrm{NW}}(\Lambda \eta, \Lambda \mathrm{x}+\mathrm{a}),
$$

and similarly for the anti-quanta NW field. This is just the hyperplane dependent analogue to the local field transformation equation,

$$
\hat{\mathrm{U}}(\Lambda, \mathrm{a}) \hat{\phi}(\mathrm{x}) \hat{\mathrm{U}}^{\dagger}(\Lambda, \mathrm{a})=\hat{\phi}(\Lambda \mathrm{x}+\mathrm{a}) .
$$

Just as the vacuum had a product state structure relative to the original NW fields at each time, so, now, the vacuum has a product state structure relative to the hyperplane generalized fields on each hyperplane. Recently (Ali, '98), in the literature of quantization over phase space and the use of positive operator valued (POV) measures for representing observables, it has also been found that relativistic systems require a form of hyperplane dependence for the dynamical variables. These investigations are driven by group theoretic considerations and the detailed relation to the more intuitively motivated considerations at work here remains to be determined.

Still, one might demur. One might say that while the construction has been rendered covariant, and we do have definite localization on each hyperplane, and the vacuum is locally empty of quanta and anti-quanta on each hyperplane when we use the generalized NW account of localization -- still, this is a counterintuitive form of localization. Localized states have a superluminal component in their evolution (no superluminal group velocities, however!) and being localized at $\mathrm{x}$ on the hyperplane of orientation $\eta_{1}$ does not require, and is, in fact, incompatible with being localized at $\mathrm{x}$ on the hyperplane with orientation $\eta_{2}$ where $\eta_{2} \neq \eta_{1}$. This is a strange kind of localization! Is there any reason to think that this is anything more than an amusing formal construction? Does generalized NW localization have any physical significance?!

To answer this question we take a classical diversion.

7. A Classical Diversion; the Center of Energy: Consider a classical system characterised, for our purposes, by a symmetric energy-momentum-stress (EMS) tensor field,

$$
\theta^{\mu \nu}(\mathrm{x})=\theta^{v \mu}(\mathrm{x}) .
$$

To begin with, we assume the system to be locally closed, i.e.

$$
\partial_{\mu} \theta^{\mu v}(\mathrm{x})=0 .
$$

In such circumstances the total 4-momentum, 


$$
\mathrm{P}^{\mu}:=\int \mathrm{d}^{3} \mathrm{x} \theta^{\mu 0}\left(\mathrm{x}^{0}, \mathbf{x}\right)
$$

is conserved, i.e. independent of the time, $x^{0}$. It is also well known that under these circumstances this same 4-momentum can be expressed as an integral over an arbitrary space-like hyperplane,

$$
\mathrm{P}^{\mu}=\int \mathrm{d}^{4} \mathrm{x} \delta(\eta \mathrm{x}-\tau) \theta^{\mu v}(\mathrm{x}) \eta_{v},
$$

where the hyperplane is composed of the points with coordinates, $\mathrm{x}$, satisfying

$$
\eta \mathrm{x}-\tau=0
$$

where $\eta$ is a future pointing, time-like, unit vector, normal to the hyperplane, and $\tau$ selects one hyperplane from all those with the same normal direction.

Clearly we could say that the total 4-momentum was hyperplane independent and that its time independence was a special case of its hyperplane independence. But this way of speaking would be appropriate only if we took seriously the association of total 4momentum with all space-like hyperplanes in every inertial frame.

To see the advantage of doing just that we consider the more general case of a system that is locally open. In other words we relax (7.2). The 4-divergence of the EMS field, $\partial_{\rho} \theta^{\mu \rho}(\mathrm{x})$, not necessarilly vanishing allows the total "4-momentum" of the system (at a definite time)

$$
\mathrm{P}^{\mu}\left(\mathrm{x}^{0}\right):=\int \mathrm{d}^{3} \mathrm{x} \theta^{\mu 0}\left(\mathrm{x}^{0}, \mathbf{x}\right)
$$

to be time dependent. As a consequence it would not, in fact, transform like a 4-vector (What would the transformed time be?). However, by generalizing the definition of the total "4-momentum" to arbitrary space-like hyperplanes, using exactly the expression on the right hand side of (7.4), we can recover a genuine 4-vector. Thus, writing

$$
\mathrm{P}^{\mu}(\eta, \tau):=\int \mathrm{d}^{4} \mathrm{x} \delta(\eta \mathrm{x}-\tau) \theta^{\mu \rho}(\mathrm{x}) \eta_{\rho}
$$

for the "4-momentum" on the $(\eta, \tau)$ hyperplane, we find that under the Poincare' transformation, $(\Lambda, a)$, we have,

$$
\mathrm{P}^{\prime \mu}\left(\eta^{\prime}, \tau^{\prime}\right)=\Lambda_{v}^{\mu} \mathrm{P}^{v}(\eta, \tau)
$$

and the quantity defined by (7.7) is a genuine 4-vector, the hyperplane dependent (HD) 4momentum of the open system. 
Similarly, (Fleming and Butterfield, '98) if we define the Center of Energy (CE) position variable, $\mathbf{X}\left(x^{0}\right)$, implicitly, by,

$$
P^{0}\left(x^{0}\right) \mathbf{X}\left(x^{0}\right):=\int d^{3} x \mathbf{x} \theta^{00}(x),
$$

then $\left(\mathrm{x}^{0}, \mathbf{X}\left(\mathrm{x}^{0}\right)\right)$ does not form a 4-vector (because of the relationship of $\mathbf{X}\left(\mathrm{x}^{0}\right)$ to all of space through the integration). But, again, we can recover a genuine 4-vector by generalizing the definition to arbitrary space-like hyperplanes,

$$
\eta P(\eta, \tau) X^{\mu}(\eta, \tau):=\int d^{4} x \delta(\eta x-\tau) x^{\mu} \theta^{\lambda \rho}(x) \eta_{\lambda} \eta_{\rho} .
$$

Under the $(\Lambda$, a) transformation we have,

$$
X^{\prime \mu}\left(\eta^{\prime}, \tau^{\prime}\right)=\Lambda_{v}^{\mu} X^{v}(\eta, \tau)+a^{\mu} .
$$

In the case of this $\mathrm{CE}$ position variable one has to generalize to hyperplanes even for locally closed systems in order to have the desired 4-vector transformation properties. The reason, of course, is that even for a closed system, unlike the total 4-momentum, the $\mathrm{CE}$ position variable is be time dependent. Quite generally, the move to hyperplanes is a generalization of time dependence which enables one to recover covariant transformation properties.

Now it turns out that this classical, physically meaningful, hyperplane dependent, position 4-vector, has properties that bear a resemblence to the counterintuitive properties of the generalized NW position eigenvectors. First, we note that $X^{\mu}(\eta, \tau)$ usually does not trace out a hyperplane independent world-line in space-time. This means that if we choose two distinct hyperplane orientations, represented by $\eta_{1}$ and $\eta_{2}$, and if the intersection of the hyperplanes $\left(\eta_{1}, \tau_{1}\right)$ and $\left(\eta_{2}, \tau_{2}\right)$ contains the point with coordinates $\mathrm{X}^{\mu}\left(\eta_{1}, \tau_{1}\right)$, it need not contain the point with coordinates $\mathrm{X}^{\mu}\left(\eta_{2}, \tau_{2}\right)$, and usually will not! The world-line traced out on the $\eta_{1}$ hyperplanes will be distinct from the world-line traced out on the $\eta_{2}$ hyperplanes. This is an obvious analogue to the failure of NW localization at the point $\mathrm{x}$ on the $\eta_{1}$ hyperplane containing $\mathrm{x}$ to imply localization at $\mathrm{x}$ on the $\eta_{2}$ hyperplane containing $\mathrm{x}$.

Second, there are circumstances, for open systems, when the hyperplane dependent world-lines of the classical CE can exhibit superluminal motion (Fig. 3.). It can happen, for instance, whenever "energy" is flowing into the system in one region and out of the system in a space-like separated region. It's a consequence of the global, collective coordinate nature of the CE. Here the analogy to NW superluminal evolution is not terribly close 


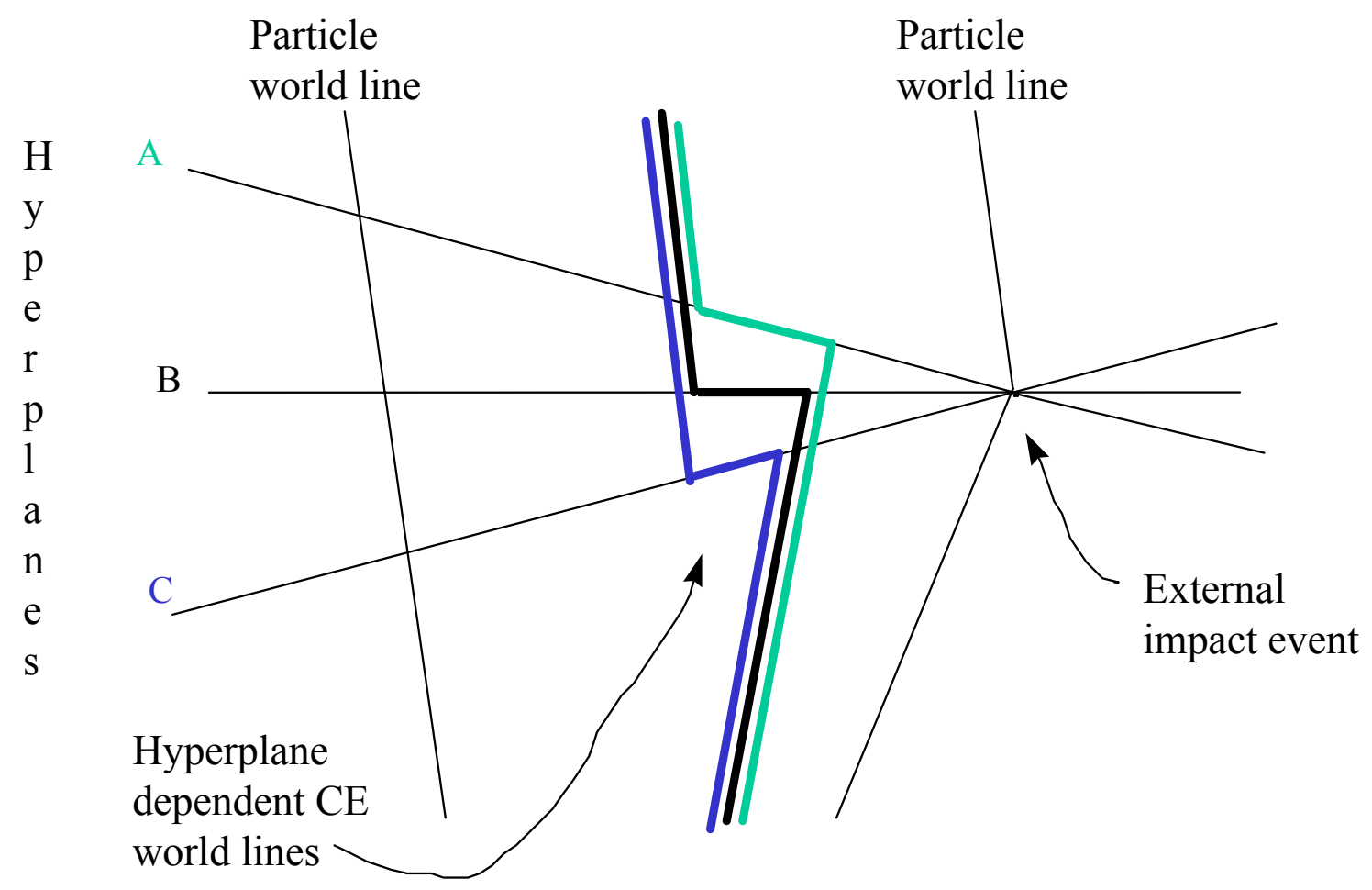

Fig. 3: Hyperplane dependence of the classical CE (open system)

At the bottom of the figure we have the world lines of two freely moving particles and the CE position variable for the two particle system. In 1+1 dimensional space-time there is no angular momentum and if we interpret this diagram in 3 space the particles must be understood to be moving directly away from each other with no perpendicular distance between their trajectories. Consequently there is no internal angular momentum. In that circumstance the $\mathrm{CE}$ position variable for the two particle system generates a hyperplane independent world line. Then the right hand particle suffers an external impact. On all those hyperplanes containing the impact event, the two particle system is open and its $\mathrm{CE}$ position variable ceases to generate an independent world line until we are beyond all the hyperplanes containing the impact event. Since the event is (unrealistically) a point event, the $\mathrm{CE}$ on hyperplanes of any fixed orientation suffers the $\mathrm{Z}$ shaped discontinuity pictured. This is due to the discontinuity in the 4momentum of the right hand particle induced by the impact. An analogous non-relativistic impact would not render discontinuous the center of mass world line since it would not change the mass of the particle. A more realistic interaction that took some time would soften the $\mathrm{Z}$ shaped $\mathrm{CE}$ world lines into $\mathrm{S}$ shaped world lines, but they might well still have superluminal portions in the middle of the $\mathrm{S}$. 


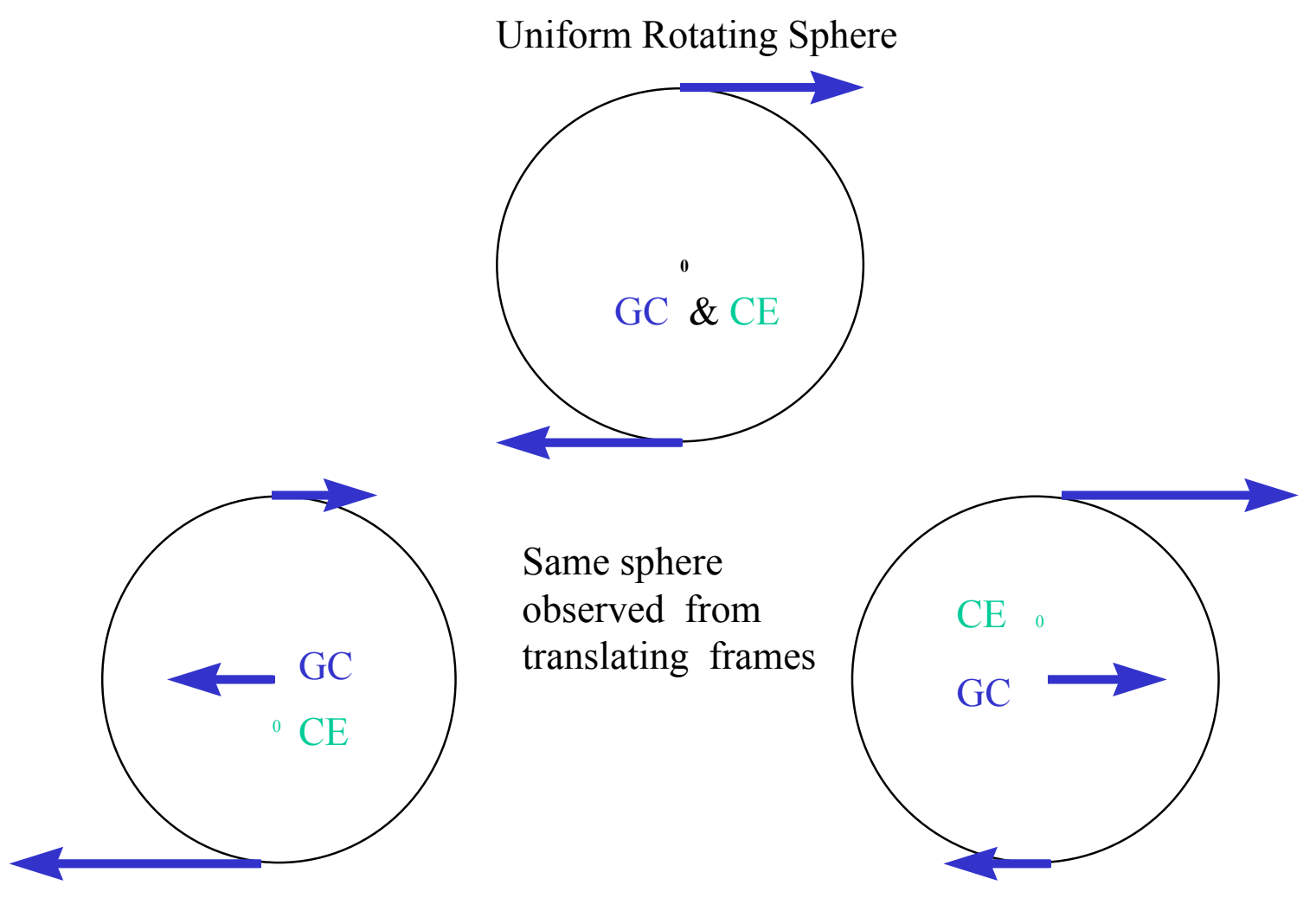

Fig. 4: Hyperplane dependence of the classical CE (rotating system)

This figure pictures the location, relative to the geometrical center, of the instantaneous $C E$ position variable of a rotating massive sphere (or cylinder) in three relatively moving inertial frames. In any single frame the same information could be conveyed by considering the $\mathrm{CE}$ position variable on three differently oriented hyperplanes, namely, hyperplanes with those orientations that made them instantaneous in the three frames respectively. This behaviour of the classical CE is replicated in the expectation values of the quantum $\mathrm{CE}$ position operator. M $\phi$ ller calculated that for the rotating Earth the CE could be as far as $\sim 10 \mathrm{~m}$. from the geometrical center in a reference frame in which the Earth was translating at nearly the speed of light. For an electron the analogous quantum calculation yields $\sim 10^{-3} \AA$. If the muon neutrino has a rest energy of $\sim 10^{-3} \mathrm{ev}$, as some recent neutrino oscillation measurements allow, the corresponding calculation yields $\sim 10^{-1} \mathrm{~mm}$ ! 
since the latter happens for closed systems as well. But the suggestion is strong that we should pursue this further.

One can rigorously show that the classical generalized CE has hyperplane dependent world-lines only for systems that are space-like extended and are open (Fig. 3.) and/or rotating (Fig. 4.). We now return to quantum field theory.

8. The CE in Quantum Field Theory: If we admit into our quantum field theory a field operator for the EMS field,

$$
\hat{\theta}^{\mu v}(\mathrm{x})=\hat{\theta}^{v \mu}(\mathrm{x}),
$$

(a step that is trivially permissable only for free fields) then we can introduce the CE position operator by analogy with the classical $\mathrm{CE}$ variable, i.e.,

$$
\eta_{v} \hat{P}^{v}: \hat{X}^{\mu}(\eta, \tau):=\int d^{4} x \delta(\eta x-\tau) x^{\mu} \hat{\theta}^{\lambda \rho}(x) \eta_{\lambda} \eta_{\rho}=\hat{M}^{\mu v} \eta_{v}+\hat{P}^{\mu} \tau .
$$

In this definition we recognize $\hat{\mathrm{P}}^{v}$ and $\hat{\mathrm{M}}^{\mu v}$ as the hermitian generators of the Poincare' group representation for the system (which exist whether the EMS field can be introduced or not - and so our CE position operator is more secure than the EMS field operator) and where the colon to the left of the position operator on the left hand side indicates a symmetrized product.

This position operator satisfies the operator constraint equation,

$$
\eta_{\mu} \hat{X}^{\mu}(\eta, \tau)=\tau,
$$

which gaurantees that the expectation values of the CE position operator will, indeed, lie on the $(\eta, \tau)$ hyperplane. It also satisfies the covariant transformation rule,

$$
\hat{\mathrm{U}}^{\dagger}(\Lambda, a) \hat{\mathrm{X}}^{\mu}\left(\eta^{\prime}, \tau^{\prime}\right) \hat{\mathrm{U}}(\Lambda, \mathrm{a})=\Lambda^{\mu}{ }_{v} \hat{\mathrm{X}}^{v}(\eta, \tau)+\mathrm{a}^{\mu},
$$

where, $\eta^{\prime}=\Lambda \eta$, and, $\tau^{\prime}=\tau+$ a $\Lambda \eta$.

But for our purposes, the most important property of the CE position operator is that,

$$
\hat{X}^{\mu}(\eta, \tau=\eta x)\left|\eta, x>_{N w}=\right| \eta, x>_{N w} x^{\mu},
$$

where,

$$
\left|\eta, \mathrm{x}>_{\mathrm{NW}}:=\hat{\phi}_{\mathrm{NW}}^{\dagger}(\eta, \mathrm{x})\right| \Omega>\text {. }
$$


In other words the field theoretic NW position eigenvectors are eigenvectors of the CE position operator! What's more, if we construct multi-quanta NW localized states such as,

$$
\left|\eta ; x_{1}, x_{2},---, x_{n}>_{N W}:=\hat{\phi}_{\mathrm{NW}}^{\dagger}\left(\eta, x_{1}\right) \hat{\phi}_{\mathrm{NW}}^{\dagger}\left(\eta, x_{2}\right)---\hat{\phi}_{\mathrm{NW}}^{\dagger}\left(\eta, \mathrm{x}_{\mathrm{n}}\right)\right| \Omega>
$$

where $\eta \mathrm{x}_{1}=\eta \mathrm{x}_{2}=---=\eta \mathrm{x}_{\mathrm{n}}$, then we find,

$$
\begin{aligned}
\eta \hat{P}: \hat{X}^{\mu}\left(\eta, \tau=\eta x_{1}\right) \mid \eta ; x_{1}, x_{2},--- & x_{n}>_{N W} \\
& =\sum_{j}\left(x_{j}{ }^{\mu}: \hbar R_{\eta}, j\right) \mid \eta ; x_{1}, x_{2},---, x_{n}>_{N W}
\end{aligned}
$$

These relations continue to hold if we include anti-quanta in the construction of the NW multi-localized state.

The NW fields are not mere formal constructions. They have unequivocal physical significance. For the quantum field theoretic system of a free scalar field, the hyperplane dependent NW scalar field, relative to which the vacuum has the structure of a product state rather than a Superentangled Reeh-Schlieder state, are fields which create and annihilate quanta and anti-quanta in states with precisely defined centers of energy. In assessing the physical significance of the Reeh-Schlieder theorem we must address the question of the comparative relevance to the actions we gross, macroscopic creatures perform, in what we judge to be bounded regions of Space-Time, of the (formal?) Minkowski coordinate variable of the local field vs. the physical Minkowski coordinate, on a hyperplane, of the NW field. For purposes of interpretation, if not for purposes of calculation, it would be foolhardy to dismiss the integral transform between these fields as immaterial to macroscopic concerns, given the global differences in the structure of the vacuum relative to them.

9. Turning on Spin and Interactions: The analysis of the structure of NW fields and the relationship of the vacuum state(s) to them in the presence of interactions remains to be carried out. The situation is complicated by the enhanced singular structure of fields in the presence of interactions. This is what originally motivated the turn to 4-dimensional smearing of the local fields in the formulation of general field theory, while our examination of the hyperplane dependent NW fields is very much a $3+1$ dimensional approach, albeit covariant. While it is hard to believe that the vacuum would become Superentangled relative to the NW fields as a consequence of turning on interactions, it is also uncertain that it would retain the product state character it displays relative to free NW fields. Furthermore, the status of the energy-momentum-stress field becomes both more tentative and more important in the presence of interactions. We leave it there for the present.

In the presence of spin, i.e. of fields with non-trivial spinorial-tensorial rank, if interactions are absent, the situation remains essentially the same as we have presented with one notable change. The corresponding hyperplane dependent NW fields can still be 
constructed and the vacuum has the product state structure relative to them, i.e. the global vacuum is a composite of local vacua. The Minkowski coordinate of the NW fields, however, is no longer the eigenvalue of the CE position operator, but of a different position operator, algebraically related to the CE. We call it the generalized NW position operator. The reason this happens is that the general CE position operator has noncommuting components for any system with non-zero internal angular momentum and, consequently, the components can not be jointly diagonalized in any basis. This is, as usual, a carry over from the non-zero Poisson brackets between the components of the classical CE for rotating systems. Happily, a Newton-Wigner position operator with commuting components continues to exist and is algebraically related to the CE position operator by, (Fleming, '65a)

$$
\hat{\mathrm{X}}_{\mathrm{NW}}{ }^{\mu}(\eta, \tau)=\hat{\mathrm{X}}_{\mathrm{CE}}{ }^{\mu}(\eta, \tau)-\frac{\varepsilon^{\mu \alpha \beta \gamma} \hat{\Sigma}_{\alpha}(\eta) \hat{\mathrm{P}}_{\beta} \eta_{\gamma}}{\hat{\mathrm{M}}(\eta \hat{\mathrm{P}}+\hat{\mathrm{M}})},
$$

where,

$$
\hat{\mathrm{M}}:=\left|\sqrt{\hat{\mathrm{P}}^{\mu} \hat{\mathrm{P}}_{\mu}}\right|,
$$

and $\quad \hat{\Sigma}^{\mu}(\eta):=(1 / 2) \varepsilon^{\mu \alpha \beta \gamma}\left\{\hat{M}_{\alpha \beta}-\hat{X}_{C E},_{\alpha}(\eta, \tau) \hat{P}_{\beta}+\hat{X}_{C E, \beta}(\eta, \tau) \hat{P}_{\alpha}\right\} \eta_{\gamma}$.

10. Conclusions: The vacuum state has the Reeh-Schlieder property relative to the local field but it does not have the Reeh-Schlieder property relative to the hyperplane dependent Newton-Wigner fields. Instead, relative to the HD-NW fields the global vacuum, on any single hyperplane, is a composite of local vacua. The HD-NW fields, and the states they create, display a counterintuitive superluminal component in their evolution, which, nevertheless is compatible with Lorentz covariance. The local fields allow the possibility of arbitrary space-like distant effects from arbitrarily localized actions, equally counterintuitive and equally compatible with Lorentz covariance! The local field evolves subluminally and satisfies microcausality but depends on a Minkowski coordinate devoid of any direct quantum physical interpretation. The HD-NW field violates microcausality but depends on a Minkowski coordinate which is related to an eigenvalue of a HD position operator, itself closely related to the classical HD center of energy position variable. How shall we choose between these perspectives?

We need not choose and we should not. Rather, wisdom lies in exploring the implications and the subtler details of the interpretation of both perspectives. To be sure, in the present context of local quantum field theory the local fields are the fundamental dynamical variables. Nevertheless, many of the HD dynamical variables and HD observables that can be constructed from the local fields represent genuine physical features of the world that local quantum field theory presents to us. Remember our discussion of the 4-momentum of an open system. Without them many questions can not be answered in a manner that explicitly displays the Lorentz covariance with which the answer must be compatible and they do have classical analogues. And in any case 
fundamentality is a variable status. Quantum field theory could, in principle, be reformulated in terms of the HD-NW fields without any reference to the local fields. I do not recommend the reformulation. The result would be much more complicated than the formulation in terms of local fields. But the integral transform from the local fields to the HD-NW fields is invertable. No information is lost thereby. The HD-NW fields could be used as the fundamental dynamical variables.

For some time I have argued that HD position operators, such as the general $\mathrm{CE}$ and the general NW position operators, are more closely related than the local field coordinate to assessments of where, on hyperplanes and in space-time, objects, systems, their localizable properties and phenomena are located. But whether this view prevails or not, the world of quantum field theory is replete with HD physical properties. Such terminology is, of course, easily misinterpreted. So let me close with the explicit declaration that for me, any quantity that can play an important role in the description and analysis of the structure and behaviour of physical systems is likely to represent a physical property of those systems.

Acknowledgements: In the work leading to this paper I have had the benefit of critical discussions with Twareque Ali, Jeremy Butterfield, Rob Clifton, Jan Hilgevoord, John Norton, Simon Saunders, Paul Teller and Andrew Wayne, none of whom are responsible for the papers' retention of my more extreme idiosyncratic views.

\section{$\underline{\text { References }}$}

S. T. Ali, (1998), "Systems of Covariance in Relativistic Quantum Mechanics", International Journal of Theoretical Physics, 37, 365 - 373.

N. D. Birrell and P. C. W. Davies, (1984), Quantum Fields in Curved Space, Cambridge Univ. Press.

R. Clifton, D. Feldman, H. Halvorson, M. L. G. Redhead, and A. Wilce, (1998), "Superentangled States," Physical Review A .

G. N. Fleming, (1965a), "Covariant Position Operators, Spin, and Locality", Physical Review, 137, B188-197.

(1965b), "Nonlocal Properties of Stable Particles", Physical Review, 139,

B963-968.

(1966), "A Manifestly Covariant Description of Arbitrary Dynamical Variables in Relativistic Quantum Mechanics", Journal of Mathematical Physics, 7, 1959-1981.

(1996), "Just How Radical is Hyperplane Dependence?", in R.Clifton (ed.), Perspectives on Quantum Reality, Dordrecht: Kluwer, pp.11-28. 
G.N. Fleming, and J. Butterfield, (1998), "Strange Positions", forthcoming in From Physics to Philosophy: Essays in Honor of Michael Redhead, Cambridge Univ. Press.

S. A. Fulling, (1989), Aspects of Quantum Field Theory in Curved Space-Time, Cambridge Univ. Press.

R. Haag, (1992), Local Quantum Physics, Springer-Verlag.

G. C. Hegerfeldt, (1985), "Violation of Causality in Relativistic Quantum Theory?", Physical Review Letters, 54, 2395-2398.

J. M. Knight, (1961), "Strict Localization in Quantum Field Theory", Journal of Mathematical Physics, 2, 459-471.

A. L. Licht, (1963), "Strict Localization", Journal of Mathematical Physics, 4, 14431447.

T. D. Newton and E. P. Wigner, (1949), "Localized States for Elementary Systems", Reviews of Modern Physics, 21, 400-406.

M. L. G. Redhead, (1995a), "More Ado About Nothing", Foundations of Physics 25: 123-137. vol. 2, 77-87.

(1995b), "The Vacuum in Relativistic Quantum Field Theory", PSA 1994,

H. Reeh and S. Schlieder, (1961) "Bemerkungen zur unitaraquivalenz von Lorentzinvarianten Feldern", Nuovo Cimento, 22: 1051

S. N. M. Ruijsenaars, (1981), "On Newton-Wigner Localization and Superluminal Propagation Speeds", Annals of Physics, 137, 33-43.

S. Saunders, (1995), "A Dissolution of the Problem of Locality", PSA 1994, vol. 2, 88-98.

W. G. Unruh, (1976), "Notes on Black Hole Evaporation", Physical Review, D14, 870.

(1990), "Particles and Fields", Quantum Mechanics in Curved Space-Time, $89-110$.

R. M. Wald, (1994), Quantum Field Theory in Curved Spacetime and Black Hole Thermodynamics, Univ. of Chicago Press.

E. P. Wigner, (1983), "Interpretation of Quantum Mechanics" in J. A. Wheeler and W. H. Zurek (eds.), Quantum Theory and Measurement, pp.260-314, Princeton Univ. Press. 
